\title{
ABÚS SEXUAL INFANTIL: CONSEQÜÈNCIES PSICOLÒGIQUES A LLARG TERMINI I FACTORS DE RISC I PROTECTORS
}

\author{
CHILD SEXUAL ABUSE: LONG-TERM \\ PSYCHOLOGICAL CONSEQUENCES \\ AND RISK FACTORS AND PROTECTIVE
}

\author{
Joana Albiñana Durà \\ Enrique Carbonell Vayä ${ }^{* *}$
}

doi: 10.7203/anuari.psicologia.15.2.33

\begin{abstract}
Resum
Objectiu: Realitzar una revisió de la literatura, per tal de comprovar les conseqüències psicològiques negatives, a llarg termini, de l'abús sexual infantil (ASI). És a dir si les víctimes d'ASI presenten una major prevalença de psicopatologies que la població general i si hi tenen algun paper altres variables, com a risc o com a mediadores. Mètode: Foren consultades quatre bases de dades mitjançant revisió sistemàtica i també es realitzà una revisió manual. Resultats i conclusions: S'inclogueren vint-i-set articles, que posen de manifest que les víctimes d'abusos sexuals infantils presenten un major nombre de psicopatologies en l'edat adulta i que, sobre aquestes, influeixen una sèrie de variables de risc que n'atenuen els efectes, mediadores, $i$ altres que els empitjoren.
\end{abstract}

Paraules clau: Abús sexual infantil, factors de risc, factors mediadors, consequiències psicològiques a llarg termini, psicopatologies en l'edat adulta.

*Universitat de València.<aldujo@alumni.uv.es>.

** Departament de Psicologia Bàsica. Facultat de Psicologia. Universitat de València. <enrique.carbonell@uv.es>.Avda. Blasco Ibáñez, 21, 46011, València, Espanya. 


\begin{abstract}
Aim: Conduct a literature review in order to ascertain the long-term negative psychological consequences of child sexual abuse (CSA), ie, if the victims of CSA showed a higher prevalence of psychopathology than the general population and whether other variables have some role, such as risk or as mediators. Method: Four databases were consulted through systematic review and also perform a manual review. Results and conclusions: Twenty-seven articles were included that highlight as victims of Child Sexual Abuse have a higher number of psychopathologies in adulthood and, on theses consequences, influence a number of variables that mitigate its effects, mediators, and other such worsening, risk variable.
\end{abstract}

Key words: Child sexual abuse, risk factors, mediating factors, psychological consequences in the long-term, psychopathology in adulthood.

\title{
Introducció
}

En el decurs de les últimes dècades, sobretot dels últims anys, s'ha pogut observar un creixent interès sobre la temàtica dels abusos sexuals infantils (ASI), cosa que s'ha vist reflectida en l'augment del nombre de recerques i estudis que s'han fet (Ruggiero, Mcleer i Dixon, 2000).

A causa dels múltiples estudis que hi ha, s'ha abordat aquesta perspectiva des de molts punts de vista i de maneres diverses, cosa que porta a l'existència de molta informació, que de vegades pot ser diferent. Un exemple d'aquests diferències pot ser la prevalença dels abusos sexuals, que ha variat en la literatura des d'un $6 \%$ a un $62 \%$ en dones, i d'un $3 \%$ a un $31 \%$ en homes (Ferguson, 1997). Per tant, es pot observar un desacord patent quant a les xifres de prevalença, però, alguns investigadors Aquesta $\mathrm{n}$ d'acord que va en augment (Pou, Jordán i Gómez, 1996). Aquesta afirmació canvia posteriorment, quan Figuero i Otero (2005) posen de manifest que realment no hi ha hagut un augment de la prevalença, sinó que s'han comunicat més casos a causa del canvi en la legislació i en el clima social. Aquestes discrepàncies es donen principalment pel que fa a les diferents definicions de l'ASI i els paràmetres inclosos (Briere i Elliot, 1994; Ferguson, 1997; Murray, Nguyen i Cohen, 2014; Wyatt i Peters, 1986). Però, a pesar de trobar-ne diferents definicions, podem destacar alguns aspectes comuns entre elles: $a$ ) Hi ha una relació desigual, per edat, maduresa o poder, entre agressor i víctima; $b$ ) Intervenen la coerció, la diferència d'edat $o$ el desenvolupament i el tipus de conducta sexual (Ferguson, 1997). A més, es troben semblances i diferències quant a l'edat límit per a l'abús sexual infantil, 
què seria una experiència abusiva, la inclusió o exclusió d'experiències amb iguals i els criteris utilitzats per a les experiències durant l'adolescència (Wyatt i Peters, 1986).

El que ha tingut una major rellevància en aquesta temàtica és l'impacte que té sobre la salut mental de les víctimes, entre les quals es presenta un major nombre de psicopatologies que entre els qui no són víctimes (Ruggiero, Mcleer i Dixon, 2000), tant a curt com a llarg termini (Calam, Horne, Glasgow i Cox, 1998). Però, a pesar d'això, una minoria d'infants que han patit abusos (d'un $10 \%$ a un $28 \%$ ) no presenten trastorns psicològics, cosa que podria ser deguda en part a les definicions de l'ASI (Briere i Elliot, 1994) i també a factors mediadors. Per això, els investigadors Aquesta $\mathrm{n}$ d'acord a afirmar que no hi ha una síndrome específica que seguisca l'ASI (Briere i Elliot, 1994; McCrae, Chapman i Christ, 2006) i que faça referència a un trastorn, a una psicopatologia específica que aparega després dels abusos sexuals infantils, sinó que és possible que n'apareguen moltes i molt diverses.

Però sí es dóna en alguns infants la síndrome d'acomodació a l'abús sexual, que consisteix a mantenir l'abús en secret, mostrar-se indefens i acomodat, revelar-lo de manera retardada, a més d'una alta probabilitat de retractar-se'n (Figuero i Otero, 2005; Weiss i Alexander, 2013). Encara que s'ha vist que un percentatge de infants víctimes d'abusos no presenten seqüeles psicològiques, es troba que les víctimes d'ASI presenten un major nombre de psicopatologies en la infantesa (Ruggiero, Mcleer i Dixon, 2000). Diferents estudis indiquen que aquests presenten majors nivells d'ansietat (Hornor, 2010; Keyes et al., 2012; Ruggiero, Mcleer i Dixon, 2000), trastorns de pànic, trastorns obsessivocompulsius (TOC) fòbies, trastorns per estrès posttraumàtic (TEPT) i depressió (Briere i Elliot, 1994; Finkelhor, 1991; Hornor, 2010; Ruggiero, Mcleer i Dixon, 2000), distorsions cognitives, com ara la culpa (Briere i Elliot, 1994) i la baixa autoestima(Briere i Elliot, 1994; Ruggiero, Mcleer i Dixon, 2000), també l'abús de substàncies (Briere i Elliot, 1994; Finkelhor, 1991; Hornor, 2010; Keyes et al., 2012), la ideació i els intents de suïcidi (Briere i Elliot, 1994; Hornor, 2010), els trastorns de comportament, el comportament antisocial (Hornor, 2010; Keyes et al., 2012), el comportament sexual indiscriminat (Briere i Elliot, 1994; Finkelhor, 1991) i la revictimització (Finkelhor, 1991).

En el desenvolupament d'aquests psicopatologies no només hi ha l'abús, sinó que alguns treballs retrospectius mostren que altres factors hi poden ser també importants (Calam, Horne, Glasgow i Cox, 1998). Algunes d'aquestes variables estan relacionades amb les característiques dels abusos com ara la severitat, la frequiència, la duració, la relació amb l'agressor, el nombre d'agressors i la violència o la força (Ruggiero, Mcleer i Dixon, 2000). També, característiques de les víctimes, com ara el gènere (Glasser i Wiseman, 2000; Ruggiero, Mcleer i Dixon, 2000) i l'edat (Ruggiero, Mcleer i Dixon, 2000). Per últim, 
hi intervenen variables relacionades amb la família, com són la reacció dels pares (Ruggiero, Mcleer i Dixon, 2000), l'incest, és a dir, el cas en què l'agressor és un familiar, majorment el pare, i els vincles afectius entre víctima i agressor (identitat), (Briere i Elliot, 1994; García-Piña, Loredo-Abdalá i Gómez-Jiménez, 2009; Glasser i Wiseman, 2000), factor molt important que cal destacar, ja que tres de cada cinc abusos es donen dins de la família i un de cada dotze el perpetra el pare o el padrastre del infant (Finkelhor, 1991), per tant, és el tipus d'abús més freqüent (García-Piña, Loredo-Abdalá i Gómez-Jiménez, 2009).

El suport dels pares també és un factor important per tal de minorar les conseqüències que pateixen els infants que han estat víctimes d'abús (Hornor, 2010; Ruggiero, Mcleer i Dixon, 2000), ja que va associat a una disminució de la simptomatologia (Briere i Elliot, 1994). Llavors, els infants que tenen un fort suport emocional després de l'abús tenen un bon pronòstic (McCrae, Chapman i Christ, 2006), i els que no en tenen ni tampoc credibilitat tenen un mal pronòstic (García-Piña, Loredo-Abdalá i Gómez-Jiménez, 2009).

Els infants víctimes d'abusos sexuals estan exposats també a problemes familiars com ara la violència domèstica, l'abús físic o l'abús de substàncies per part dels pares (McCrae, Chapman i Christ, 2006). A més, els diferents tipus de maltractaments, com l'abús físic, l'emocional i la negligència es correlacionen i ocorren alhora, cosa que pot agreujar també el desenvolupament i el pronòstic (Keyes et al., 2012). Per tant, és possible observar que l'abús sexual infantil pot comportar problemes psicològics greus en la infantesa, encara que pot haver-hi factors que l'atenuen. Però quan es fa referència a les conseqüències que apareixen en l'edat adulta s'ha de tenir en compte que una reacció comportamental o emocional forta després de l'abús sexual no significa que persistisca a llarg termini, i tampoc l'absència de símptomes en un primer moment significa que no apareguen posteriorment (McCrae, Chapman i Christ, 2006). Per això, cal destacar que aproximadament un 30\% dels infants que han patit abusos sexuals en la infantesa patirà conseqüències psicològiques a llarg termini (García-Piña, Loredo-Abdalá i Gómez-Jiménez, 2009).

És de gran rellevància el diagnòstic primerenc dels ASI, encara que és molt complicat fer-lo, ja que els indicis físics, són quasi inexistents (Pou, Jordán i Gómez, 1996). A més, no sol haver-hi testimonis que no siguen les víctimes o els agressors, per això moltes vegades els professionals els basen en els testimonis dels infants (Berlinerblau i Taylor, 2002; Redondo i Ortiz, 2005). Per fer un diagnòstic correcte s'entrevisten els cuidadors, es fa una exploració física, exàmens de laboratori i una avaluació psicològica o psiquiàtrica, tant del infant com del cuidador (García-Piña, Loredo-Abdalá i Gómez-Jiménez, 2009). És de gran importància el tractament de les víctimes i els familiars a través d'un equip multidisciplinari per ajudar-los en l'acceptació de l'abús i alleujar els sentiments de ràbia i de culpa, a més de tractar l'ansietat, la depressió i altres psi- 
copatologies que puguen sorgir (Echeburúa i Guerricaechevarria, 2011), en el qual cal incloure un assistent social, un pediatre, una infermera, un policia i un psicòleg per fer un diagnòstic correcte i per a la prevenció (Pou, Jordán i Gómez, 1996; Redondo i Ortiz, 2005).

Si no es fa un diagnòstic, un tractament o no es tenen elements de suport intrafamiliars, com ja s'ha vist, un 30\% d'aquestes víctimes poden presentar problemes psicològics en l'edat adulta (García-Piña, Loredo-Abdalá i GómezJiménez, 2009).

\section{Objectiu}

Revisar la literatura dels últims anys sobre els abusos sexuals infantils i les conseqüències psicopatològiques que se'n poden derivar i que presenten les víctimes en l'edat adulta, com ara l'ansietat, la depressió, el trastorn per estrès posttraumàtic, la ideació i els intents de suïcidi i l'abús de substàncies, considerant que hi haurà una major presència d'aquests entre les víctimes d'abusos, que entre els qui no són víctimes. A més, les psicopatologies que es presenten en l'edat adulta són similars a les que presenten les víctimes d'ASI en la infantesa. S'estudiaran també si hi ha altres factors que poden influir, d'una banda, agreujant les consequiències, com ara els de risc, i d'una altra, atenuant els símptomes i les conseqüències psicològiques o psicopatològiques que se'n poden derivar, com ara els factors mediadors.

\section{Metodologia}

La metodologia per a realitzar aquest estudi ha estat la revisió de la literatura publicada, des de l'any 2000, sobre la temàtica que es mostra.

Han estat emprades dues tècniques per a fer els estudis rellevants, que després seran inclosos en la revisió. En primer lloc, s'ha fet la revisió sistemàtica mitjançant bases de dades en Internet i, en segon lloc, s'ha utilitzat també la revisió manual.

Les dades de bases emprades per a realitzar la cerca han estat quatre: PubMed, Dialnet, ScienceDirect i PsycINFO. En aquests bases de dades s'han buscat articles compresos entre l'any 2000 fins l'actualitat (2014), s'han emprat paraules claus relacionades amb la temàtica del treball. La resta foren identificats per revisió manual de la literatura dels articles rellevants que s'han obtingut, a partir de la revisió sistemàtica prèvia.

Per tal de considerar un estudi adequat per a la seua inclusió en la present revisió de la literatura, havia de complir una sèrie de criteris d'inclusió, els quals han estat: 1. Datats a partir de l'any 2000; 2. L'ASI; 3. Les psicopatologies en l'edat adulta; 4. Incloure factors mediadors i/o de risc entre l'abús sexual 
infantil i les psicopatologies; 5. Ser una revisió de la bibliografia, estudis empírics o un estudi de casos i 6. En l'últim cas (estudi de casos), presentar anàlisis estadístiques. Aquests criteris d'inclusió foren aplicats a tots els articles, atès el seu interès per a la temàtica del treball i per la qualitat que presentava.

Una vegada seleccionats els articles que s'inclourien en l'estudi de revisió de la literatura, s'extrauen una sèrie de dades dels diferents estudis: 1. Característiques de l'estudi: nom de l'estudi, autors, any, revista i lloc de publicació; 2. Població estudiada: nombre de participants, gènere i edat; 3 . Psicopatologies desenvolupades; 4. Mètode de selecció i d'avaluació; 5 . Presència de mediadors i/o variables de risc.

Per a l'obtenció de la literatura per a la realització de la següent revisió s'han seguit tres passos bàsics: cerca inicial, cerca sistemàtica i cerca manual. En primer lloc, la cerca inicial s'ha fet per veure l'estat de la qüestió, el volum i el tipus d'estudis que hi ha i quines són les bases de dades que es poden utilitzar i que resultarien més útils, i s'ha pogut comprovar que el volum d'estudis sobre els abusos sexuals infantils és d'una magnitud important, la qual cosa indica la rellevància de la qüestió. Partint de la revisió sistemàtica i utilitzant les bases de dades, PubMed, Dialnet, ScienceDirect i PsycINFO, varen ser identificats 27.035 articles. Després de llegir el resum i l'article complet i de descartar els que estaven duplicats o no complien els criteris, es van obtenir divuit articles per a incloure'ls en la revisió de la literatura.

Quant a la revisió manual, es va seguir un procediment similar. A partir de la bibliografia dels articles més rellevants de la revisió sistemàtica s'identificaren 95 articles, dels quals, seguint el procediment anterior, se'n seleccionaren nou.

\section{Resultats}

Els resultats que s'han obtingut dels vint-i-set articles inclosos en aquesta revisió de la literatura inclouen, com es pot veure en la taula 1, el primer autor de l'article, el país on ha estat realitzat, la revista o lloc de publicació, els participants -nom, gènere i edat-, les psicopatologies que s'avaluen, els mètodes i els instruments d'avaluació, factors influents, com ara, d'una banda, els mediadors, i d'una altra, les variables de risc, i per últim la prevalença de l'abús sexual infantil. En aquesta taula han estat classificats els articles i citats, perquè siga més senzilla la comprovació de la bibliografia.

La majoria d'estudis que s'han inclòs en aquesta revisió procedeixen d'Estats Units, un total de nou estudis. Després, d'on més se n'han obtingut ha estat d'Espanya, amb un nombre de quatre, a l'igual que els estudis provinents d'Austràlia. Els altres països que han aportat articles, han estat el Regne Unit, Canadà, Nova Zelanda, Suïssa i Itàlia. Per tant, s'hi han inclòs articles de vuit països diferents, com es pot veure en la taula 1 . 
Es pot observar també que hi ha una gran varietat de publicacions diferents. La revista on més estudis dels inclosos han aparegut publicats ha estat Child Abuse and Neglect, amb nou estudis. Dos articles publicats a The British Journal of Psychiatry, a l'igual que de l'International Journal of Offender Therapy and Comparative Criminology. Dos articles més en la revista Archives of General Psychiatry. D'altres publicacions només s'inclogué un article: Clinical Psychology and Psychotherapy, Clinical Psychology Review, Papeles del Psicólogo, Psicothema, International Journal of Public Health, un altre de Comprehensive Psychiatry, Trauma, Violence and Abuse, Journal of Clinical Psychiatry, American Journal of Public Health, Psychological Medicine, Agression and Violent Behavior i Journal of Interpersonal, de manera que hi ha setze publicacions diferents.

El tipus de disseny que més ha estat utilitzat fou el disseny retrospectiu, que s'ha emprat en vuit estudis. Després, la metodologia més emprada estigué la revisió de la literatura, feta servir en cinc estudis. Amb disseny longitudinal s'han utilitzat quatre articles. També s'han inclòs tres estudis prospectius, tres estudis amb bessons i tres de disseny comunitari. Finalment, també un estudi amb disseny de múltiples etapes amb mostreig probabilístic estratificat. Les referències dels quals poden comprovar-se en la taula 1.

Les mostres dels estudis varien quant al nombre. Van de quaranta-quatre subjectes en un estudi realitzat per Dimitrova et al. (2010), a un estudi realitzat per Pérez-Fuentes et al. (2013), en el qual inclou 34.217 subjectes. Per tant, hi ha una gran varietat quant al nombre de mostres. Quant al gènere dels subjectes que s'han utilitzat per realitzar cada estudi, es pot trobar que la majoria d'estudis, en concret quinze, utilitzen mostres que comprenen ambdós sexes, encara que sol haver-hi més percentatge de dones que d'homes, segueixen en quantitat d'estudis, sis, els que inclouen només dones en la mostra emprada. I finalment, s'han estudiat dos treballs que només inclouen homes en les mostres. De nou, la bibliografia i classificació es mostra en la taula 1.

Quant a la prevalença d'abusos sexuals que hi ha en cada mostra, cal ressaltar que molts dels estudis no parlen d'aquest problema, ja que són de vegades mostres expressament seleccionades, en què se sap prèviament que presenten abusos sexuals infantils. Però els estudis on sí apareix un percentatge, varia considerablement entre mostres en què es troben uns percentatges que van des d'un $10 \%$ a un $15 \%$ (Romano i De Luca, 2001) fins a percentatges que van del $18 \%$ al 63\% (Steel, Sanna, Hammond, Whipple i Cross, 2004), però sobre tot, entre gèneres. La prevalença varia quant al gènere femení, d'un 5,9\% de la mostra (Dinwiddie, et al., 2000) a un 41,6\% (Young, Harford, Kinder i Savell, 2007). Pel que fa al gènere masculí, els percentatges també varien, encara que en tots els estudis aquests són menors. Van d'un 2,5\% dels participants (Dinwiddie, et al., 2000; Molnar, Buka i Kessler, 2001), fins un 30,7\% (Young, Harford, Kinder i Savell, 2007). 
TAULA 1

Classificació dels estudis segons les seues característiques.

\begin{tabular}{|c|c|c|}
\hline \multirow[t]{8}{*}{ País } & Estats Units (9) & $\begin{array}{l}\text { Briere, et al. (2003); Cutajar et al. (2010); Fassler, } \\
\text { et al. (2005); Johnson et al. (2011); Kendler et al. } \\
\text { (2000); Pérez-Fuentes et al. (2013); Sperry et al. } \\
\text { (2013); Steel et al. (2004); Young et al. (2007) }\end{array}$ \\
\hline & Espanya (4) & $\begin{array}{l}\text { Cantón et al. (2008); Cantón et al. (2010); Pareda } \\
\text { (2010); Villagrá et al. (2013) }\end{array}$ \\
\hline & Austràlia (4) & $\begin{array}{l}\text { Cutajar et al. (2010); Cutajar et al. (2010); Dinwiddie } \\
\text { et al. (2000); Spataro et al. (2004) }\end{array}$ \\
\hline & Regne Unit (3) & Bulik et al. (2001); Chou (2012); Hillberg et al. (2011) \\
\hline & Canadà (2) & Forouzan et al. (2005); Romano et al. (2001) \\
\hline & Nova Zelanda (2) & Fergusson et al. (2008); Fergusson et al. (2013) \\
\hline & Suïssa (2) & Dimitrova et al. (2010); Lindert et al. (2013) \\
\hline & Itàlia (1) & Maniglio (2009) \\
\hline \multirow[t]{10}{*}{ Publicació } & $\begin{array}{l}\text { Child Abuse and } \\
\text { Neglect (9) }\end{array}$ & $\begin{array}{l}\text { Briere et al. (2003); Cantón et al. (2010); Cutajar et } \\
\text { al. (2010); Fassler et al. (2005); Fergusson et al. } \\
\text { (2008); Fergusson et al. (2013); Johnson et al. } \\
\text { (2011); Sperry et al. (2013); Steel et al. (2004) }\end{array}$ \\
\hline & $\begin{array}{l}\text { The British Journal } \\
\text { of Psychiatry (2) }\end{array}$ & Bulik et al. (2001); Spataro et al. (2004) \\
\hline & $\begin{array}{l}\text { International Journal } \\
\text { of Offender Therapy } \\
\text { and Comparative } \\
\text { Criminology (2) }\end{array}$ & Forouzan et al. (2005); Villagrá et al. (2013) \\
\hline & $\begin{array}{l}\text { Archives of General } \\
\text { Psychiatry (2) }\end{array}$ & Cutajar et al. (2010); Kendler et al. (2000) \\
\hline & $\begin{array}{l}\text { Clinical Psychology } \\
\text { and Psychotherapy (1) } \\
\text { Clinical Psychology } \\
\text { Review (1) }\end{array}$ & $\begin{array}{l}\text { Dimitrova et al.(2010) } \\
\text { Maniglio (2009) }\end{array}$ \\
\hline & $\begin{array}{l}\text { Papeles del Psicólogo } \\
\text { (1) }\end{array}$ & Pareda (2010) \\
\hline & Psicothema (1) & Cantón et al. (2008) \\
\hline & $\begin{array}{l}\text { International Journal } \\
\text { of Public Health (1) }\end{array}$ & Lindert et al. (2013) \\
\hline & $\begin{array}{l}\text { Comprehensive } \\
\text { Psychiatry (1) }\end{array}$ & Pérez-Fuentes et al. (2013) \\
\hline & $\begin{array}{l}\text { Trauma, Violence } \\
\text { and Abuse (1) }\end{array}$ & Hillberg et al. (2011) \\
\hline
\end{tabular}




\begin{tabular}{|c|c|c|}
\hline \multirow[t]{5}{*}{ Publicació } & $\begin{array}{l}\text { Journal of Clinical } \\
\text { Psychiatry (1) }\end{array}$ & Chou (2012) \\
\hline & $\begin{array}{l}\text { American Journal } \\
\text { of Public Health (1) }\end{array}$ & Molnar et al. (2001) \\
\hline & $\begin{array}{l}\text { Psychological } \\
\text { Medicine (1) }\end{array}$ & Dinwiddie et al. (2000) \\
\hline & $\begin{array}{l}\text { Agression and Violent } \\
\text { Behavior (1) }\end{array}$ & Romano et al. (2001) \\
\hline & $\begin{array}{l}\text { Journal of Interperso- } \\
\text { nal (1) }\end{array}$ & Young et al. (2007) \\
\hline \multirow[t]{7}{*}{ Disseny } & Retrospectiu (8) & $\begin{array}{l}\text { Briere et al. (2003); Cantón et al. (2010); Dimitrova } \\
\text { et al. (2010); Forouzan et al. (2005); Johnson et al. } \\
\text { (2011); Steel et al. (2004); Villagrá et al. (2013); } \\
\text { Young et al. (2007) }\end{array}$ \\
\hline & $\begin{array}{l}\text { Revisió de la litera- } \\
\text { tura }(5)\end{array}$ & $\begin{array}{l}\text { Hillberg et al. (2011); Lindert et al. (2013); Maniglio } \\
\text { (2009); Pareda (2010); Romano et al. (2001) }\end{array}$ \\
\hline & Longitudinal (4) & $\begin{array}{l}\text { Cutajar et al. (2010); Cutajar et al. (2010); Fergusson } \\
\text { et al. (2008); Fergusson et al. (2013) }\end{array}$ \\
\hline & Prospectiu (3) & $\begin{array}{l}\text { Cantón et al. (2008); Spataro et al. (2004); Sperry et } \\
\text { al. (2013) }\end{array}$ \\
\hline & $\begin{array}{l}\text { Nacional o comunitari } \\
\text { (3) }\end{array}$ & $\begin{array}{l}\text { Bulik et al. (2001); Dinwiddie et al. (2000); Kendler } \\
\text { et al. (2000) }\end{array}$ \\
\hline & $\begin{array}{l}\text { Estudi amb bessons } \\
\text { (3) }\end{array}$ & $\begin{array}{l}\text { Fassler et al. (2005); Molnar et al. (2001); Pérez- } \\
\text { Fuentes et al. (2013) }\end{array}$ \\
\hline & $\begin{array}{l}\text { Mostreig prob. estra- } \\
\text { tificat (1) }\end{array}$ & Chou (2012) \\
\hline \multirow[t]{3}{*}{ Mostra } & $\begin{array}{l}\text { Mixta (homes i dones) } \\
\text { (15) }\end{array}$ & $\begin{array}{l}\text { Briere et al. (2003); Cantón et al. (2008); Cantón et } \\
\text { al. (2010); Chou (2012); Cutajar et al. (2010); Cuta- } \\
\text { jar et al. (2010); Fergusson et al. (2008); Fergusson } \\
\text { et al. (2013); Molnar et al. (2001); Pérez-Fuentes et } \\
\text { al. (2013); Spataro et al. (2004); Sperry et al. (2013); } \\
\text { Steel et al. (2004); Villagrá et al. (2013); Young et al. } \\
\text { (2007) }\end{array}$ \\
\hline & Dones (6) & $\begin{array}{l}\text { Bulik et al. (2001); Dimitrova et al. (2010); Dinwid- } \\
\text { die et al. (2000); Fassler et al. (2005); Johnson et al. } \\
\text { (2011); Kendler et al. (2000); }\end{array}$ \\
\hline & Homes (2) & Forouzan et al. (2005); Romano et al. (2001) \\
\hline $\begin{array}{l}\text { Mètode } \\
\text { Selecció } \\
\text { Mostra }\end{array}$ & $\begin{array}{l}\text { Entrevista cara a cara } \\
(11)\end{array}$ & $\begin{array}{l}\text { Bulik et al. (2001); Chou (2012); Dimitrova et al. } \\
\text { (2010); Fassler et al. (2005); Fergusson et al. (2008); } \\
\text { Fergusson et al (2013); Johnson et al. (2011); Kend- } \\
\text { ler et al. (2000); Pérez-Fuentes et al. (2013); Sperry } \\
\text { et al. (2013); Villagrá et al. (2013) }\end{array}$ \\
\hline
\end{tabular}




\begin{tabular}{|c|c|c|}
\hline \multirow{3}{*}{$\begin{array}{l}\text { Mètode } \\
\text { Selecció } \\
\text { Mostra }\end{array}$} & $\begin{array}{l}\text { Qüestionaris via } \\
\text { correu electrònic (4) }\end{array}$ & $\begin{array}{l}\text { Briere et al. (2003); Bulik et al. (2001); Chou (2012); } \\
\text { Dinwiddie et al. (2000) }\end{array}$ \\
\hline & Registres oficials & $\begin{array}{l}\text { Bulik et al. (2001); Cutajar et al. (2010); Molnar et } \\
\text { al. (2001); Spataro et al. (2004); Steel et al. (2004) }\end{array}$ \\
\hline & $\begin{array}{l}\text { Entrevistes telefòni- } \\
\text { ques (2) }\end{array}$ & Dinwiddie et al. (2000); Johnson et al. (2011) \\
\hline \multirow[t]{6}{*}{$\begin{array}{l}\text { Psicopato- } \\
\text { logies }\end{array}$} & Ansietat (19) & $\begin{array}{l}\text { Briere et al. (2003); Bulik et al. (2001); Chou et al. } \\
\text { (2012); Cutajar et al. (2010); Dimitrova, et al. (2010); } \\
\text { Dinwiddie et al. (2000); Fergusson et al. (2008); Fer- } \\
\text { gusson et al. (2013); Hillberg et al. (2011); Kendler } \\
\text { et al. (2000); Lindert et al. (2013); Molnar et al. } \\
\text { (2001); Pérez-Fuentes et al. (2013); Romano et al. } \\
\text { (2001); Spataro et al. (2004); Sperry et al. (2013); } \\
\text { Steel et al. (2004); Young et al. (2007) }\end{array}$ \\
\hline & Depressió (18) & $\begin{array}{l}\text { Briere et al. (2003); Bulik et al. (2001); Cantón et al. } \\
\text { (2008); Chou et al. (2012); Dinwiddie et al. (2000); } \\
\text { Fergusson et al. (2008); Fergusson et al. (2013); Fo- } \\
\text { rouzan et al. (2005); Hillberg et al. (2011); Johnson } \\
\text { et al. (2011); Kendler et al. (2000); Lindert et al. } \\
\text { (2013); Molnar et al. (2001); Pérez-Fuentes et al. } \\
\text { (2013); Romano et al. (2001); Sperry et al. (2013); } \\
\text { Steel et al. (2004); Young et al. (2007) }\end{array}$ \\
\hline & $\begin{array}{l}\text { Abús d'alcohol } \\
\text { i drogues (16) }\end{array}$ & $\begin{array}{l}\text { Bulik et al. (2001); Chou et al. (2012); Cutajar et al. } \\
\text { (2010); Dinwiddie et al. (2000); Fassler et al. (2005); } \\
\text { Fergusson et al. (2008); Fergusson et al. (2013); Fo- } \\
\text { rouzan et al. (2005); Hillberg et al. (2011); Kendler } \\
\text { et al. (2000); Molnar et al. (2001); Pérez-Fuentes et } \\
\text { al. (2013); Romano et al. (2001); Spataro et al. (2004); } \\
\text { Sperry et al. (2013); Villagrá et al. (2013) }\end{array}$ \\
\hline & $\begin{array}{l}\text { Trastorn per estrès } \\
\text { posttraumàtic (9) }\end{array}$ & $\begin{array}{l}\text { Chou et al. (2012); Cantón et al. (2010); Cutajar et } \\
\text { al. (2010); Cutajar et al. (2010); Fergusson et al. } \\
\text { (2013); Hillberg et al. (2011); Johnson et al. (2011); } \\
\text { Molnar et al. (2001); Pareda (2010); Pérez-Fuentes } \\
\text { et al. (2013) }\end{array}$ \\
\hline & Trastorns psicòtics (8) & $\begin{array}{l}\text { Cutajar et al. (2010); Cutajar et al. (2010); Forouzan } \\
\text { et al. (2005); Hillberg et al. (2011); Pérez-Fuentes et } \\
\text { al. (2013); Spataro et al. (2004); Steel et al. (2004); } \\
\text { Young et al. (2007) }\end{array}$ \\
\hline & $\begin{array}{l}\text { Ideació i intents } \\
\text { de suïcidi (6) }\end{array}$ & $\begin{array}{l}\text { Chou et al. (2012); Dinwiddie et al. (2000); Fergus- } \\
\text { son et al. (2008); Fergusson et al. (2013); Hillberg et } \\
\text { al. (2011); Pérez-Fuentes et al. (2013) }\end{array}$ \\
\hline
\end{tabular}




\begin{tabular}{|c|c|c|c|}
\hline \multirow[t]{12}{*}{$\begin{array}{l}\text { Mediadors/ } \\
\text { variables } \\
\text { de risc }\end{array}$} & \multirow[t]{6}{*}{$\begin{array}{l}\text { Media- } \\
\text { dors }\end{array}$} & $\begin{array}{l}\text { Relacions } \\
\text { properes } \\
\text { i d'aferrament }\end{array}$ & Dimitrova et al. (2010); Forouzan et al. (2005) \\
\hline & & $\begin{array}{l}\text { Suport social/ } \\
\text { familiar }\end{array}$ & Forouzan et al. (2005); Sperry et al. (2013) \\
\hline & & $\begin{array}{l}\text { Estratègies } \\
\text { d'afrontament }\end{array}$ & $\begin{array}{l}\text { Cantón et al. (2008); Cantón et al. (2010); Steel } \\
\text { et al. (2004) }\end{array}$ \\
\hline & & $\begin{array}{l}\text { Funcionament } \\
\text { familiar positiu }\end{array}$ & Kendler et al. (2000) \\
\hline & & Gènere & Chou (2012); Young et al. (2007) \\
\hline & & $\begin{array}{l}\text { Comunicació } \\
\text { de l'abús }\end{array}$ & Bulik et al. (2001) \\
\hline & \multirow[t]{6}{*}{ V. risc } & $\begin{array}{l}\text { Característiques } \\
\text { de l'abús }\end{array}$ & $\begin{array}{l}\text { Briere et al. (2003); Bulik et al. (2001); Cantón } \\
\text { et al. (2010); Cutajar et al. (2010); Fassler et al. } \\
\text { (2005); Hillberg et al. (2011); Johnson et al. } \\
\text { (2011); Molnar et al. (2001); Romano et al. (2001); } \\
\text { Steel et al. (2004) }\end{array}$ \\
\hline & & $\begin{array}{l}\text { Característiques } \\
\text { sociodemogràfi- } \\
\text { ques }\end{array}$ & $\begin{array}{l}\text { Briere et al. (2003); Chou et al. (2012); Fergusson } \\
\text { et al. (2013); Pérez-Fuentes et al. (2013); Sperry } \\
\text { et al. (2013) }\end{array}$ \\
\hline & & $\begin{array}{l}\text { Funcionament } \\
\text { familiar negatiu }\end{array}$ & $\begin{array}{l}\text { Fassler et al. (2005); Fergusson et al. (2008); } \\
\text { Fergusson et al. (2013); Hillberg et al. (2011) }\end{array}$ \\
\hline & & Gènere & $\begin{array}{l}\text { Fergusson et al. (2008); Hillberg et al. (2011); } \\
\text { Villagrá et al. (2013) }\end{array}$ \\
\hline & & $\begin{array}{l}\text { Mesures socio- } \\
\text { econòmiques } \\
\text { familiars }\end{array}$ & Fergusson et al. (2008) \\
\hline & & $\begin{array}{l}\text { Psicopatologies } \\
\text { parentals }\end{array}$ & Dinwiddie et al. (2000); Kendler et al. (2000) \\
\hline
\end{tabular}

Pel que fa als mètodes de selecció de la mostra, també difereixen. Es pot ressaltar l'entrevista cara a cara amb els possibles subjectes dels estudis com a mètode més emprat, utilitzat en onze treballs. Els altres mètodes que s'han fet servir en els diferents estudis han estat els registres oficials, d'institucions psiquiàtriques o hospitals en cinc treballs, qüestionaris via correu electrònic, en quatre estudis, i les entrevistes telefòniques en dos estudis. També es pot consultar la bibliografia en la taula 1 .

Múltiples estudis, a l'hora d'avaluar les psicopatologies que volen estudiar, utilitzen instruments i escales generals, sense centrar-se en cap trastorn mental en concret, com són els qüestionaris basats en el CIDI (OMS, 1993), entrevistes del DSM-IV (APA, 1994), Global Assessment of Functioning (GAF), escala del 
DSM-IV (APA, 1994), Structured Clinical Interview for DSM-IV (SCIDI-I/P; First, Spitzer, Gibbon i Williams, 1996), Clinical Interview Schedule (Alaya, Lewis, Perosi, 1992), Mini International Neuropsychiatric Interview (MINI; versió espanyola 5.0 de Ferrando et al., 2000), Millon Clinical Multiaxial Inventory-I (MCMI-I; Millon, 1983), Symptom Checklist 90-R (SCL-90-R; Derogatis, 1977; SCL-90-R; Derogatis, 1983), Structured Clinical Interview DSM-III$R$ (Spitzer et al., 1998), SSGA (Bucholz et al., 1994), Brief Symptom Inventory (BSI; Derogatis, 1993).

Les psicopatologies més estudiades foren, en primer lloc, l'ansietat, de la qual parlen dinou estudis. L'ansietat apareix avaluada per diferents qüestionaris, com The Beck Anxiety Inventory (BAI; Beck i Steer, 1990). En segon lloc, trobem la depressió, amb divuit treballs, aquesta és avaluada per diferents instruments: Center of Epidemiological Studies of Depression Scale (CES-D; Radloff, 1977), Beck Depression Inventory (BDI; Beck, Ward, Mendelson, Mock i Erbaugh, adaptada per Conde, Aquestban i Useros, 1976), Beck Depression Inventory-II (Beck, Steer i Brown, 1996) i Depressed Mood Index (Moos, Crontike, Billings i Finnew, 1984). Després, trobem també l'abús de drogues i d'alcohol, amb setze estudis. L'abús de substàncies era també avaluat per diferents instruments, com el Rutgers Health and Human Development Project (Pandina, Labouvie i White, 1984), Alcohol Use Disorder Identification Test (Aasland, Babor i Saunders, 1993), Severity of Alcohol Dependence Questionnaire (McGrath, Sitharthan, Stockwell et al., 1994), Clinical Interview Schedule (Alaya, Lewis, Perosi et al., 1992) i Addiction Severity Index-6 (ASI-6; Adaptació espanyola de Bobes et al., 2007). Seguidament, apareixen nou estudis que avaluen el trastorn per estrès posttraumàtic, que és avaluat per instruments com ara Diagnostic Interview Schedule (DIS; Schulman, Scharf, Lumer i Maurer, 2001), Clinician Administered PTDS Scale (CAPS; Blacke et al., 1996), Trauma Screening Questionaire (Andrews, Brewin i Rose, 2002), Escala de Gravedad de Síntomas de Estrés Postraumático (Severity Symptoms of PTDS Scale; Echeburúa, Corral, Amor, Zubizarreta i Sarasua, 1997) i el Trauma Symptom Inventory (TSI; Briere, 1997). Trobem el mateix nombre d'articles que avaluen els trastorns psicòtics. Finalment, cal destacar l'avaluació de la ideació suïcida i dels intents de suïcidi per sis articles.

Els trastorns mentals que acaben de ser citats foren els més estudiats pels diferents treballs, encara que se n'han avaluat altres com per exemple el trastorn de personalitat límit (TLP) (Cutajar et al., 2010; Forouzan i Van Gijseghem, 2005; Hillberg, Hamilton i Dixon, 2011; Pareda, 2010), els trastorns dissociatius en un estudi de Johnson, Pike i Chard (2011), els trastorns alimentaris, estudiats per Chou (2012), o el trastorn de personalitat antisocial (Fergusson, Boden i Horwood, 2008). Amb referència a l'abús sexual infantil, aquest era avaluat en la majoria d'estudis per qüestionaris, (Briere i Elliott, 2003; Cantón 
i Cantón, 2010; Cantón i Justicia, 2008; Dimitrova et al., 2010; Kendler et al., 2000; Pérez-Fuentes et al., 2013; Steel, Sanna, Hammond, Whipple i Cross, 2004; Young, Harford, Kinder i Savell, 2007), en els quals s'informava sobre característiques de l'abús, de la víctima i l'agressor. Altres estudis empraven l'entrevista, com ara l'estudi de Fergusson, Boden i Horwood (2008), un altre de Fergusson, McLeod i Horwood (2013), i per últim un de Johnson, Pike i Chard (2011), entre les quals s'inclou la Trauma Interview for sexual abuse victims (Resick, Jordan Girelli i Hutter, 1988). Finalment, un dels estudis, realitzat per Fassler, Amodeo, Griffin, Clay i Ellis (2005) utilitzava un mètode mixt, amb qüestionaris i entrevista. Pel que fa als qüestionaris, se'n poden destacar dos que foren emprats en algun estudi: l'Early Trauma Inventory (ETI; Bremner, Vermetten i Mazure, 2000) i el Traumatic Events Survey (TES; Briere, 1997).

Per últim, cal duestacar la presència en diversos estudis, d'una banda, de factors mediadors, i d'una altra, de variables de risc. En primer lloc, quant als mediadors, trobem estudis de Dimitrova et al. (2010) i de Forouzan i Van Gijseghem (2005), que parlen de les relacions properes i d'aferrament, que s'avalua amb l'Adult Attatchment Scale (AAS; Colluns i Read, 1990) i el Relationship Questionaire (Bartholomew i Horowitz, 1991). També es troba com a mediador el suport social i el familiar, que s'avalua amb la Interpersonal Support Evaluation List (ISEL; Cohen, Mermelstein, Kamarck i Hoberman, 1983). Les estratègies d'afrontament són estudiades per Cantón i Justicia (2008), Cantón i Cantón (2010) i Steel, Sanna, Hammond, Whipple i Cross (2004), i són avaluades per la How I deal with things Scale (Burt i Katz, 1987) i la Ways of Coping Checklist-R (Lazarus i Folkman, 1984). Per últim, s'estudia també el funcionament familiar positiu, el gènere i la comunicació de l'abús. En segon lloc, pel que fa als factors de risc, es troba que l'aspecte més estudiat és el de les característiques de l'abús, i seguidament les característiques sociodemogràtiques. També s'estudia el funcionament familiar negatiu, avaluat amb la Family Environment Scale (FES; Moos i Moos, 1981), el gènere, les mesures socioeconòmiques familiars i les psicopatologies parentals. Tots els estudis que inclouen la informació que acabem de citar es poden trobar en la taula 1, amb les corresponents referències.

\section{Discussió}

Per fer aquest estudi de revisió de la literatura, sobre els abusos sexuals infantils i les psicopatologies en què aquests es poden desenvolupar en l'edat adulta, s'han emprat vint-i-set articles. S'ha comprovat que, com ja s'ha dit, és un problema de gran rellevància, perquè apareix molta bibliografia al respecte, a més de ser de diversa procedència i aparèixer publicada en diverses revistes diferents. 
S'ha pogut observar que l'abús sexual infantil comporta nombrosos problemes de salut mental a llarg termini. Aquestes víctimes presenten majors nivells d'ansietat, com es pot veure en els estudis de Cutajar et al. (2010), Maniglio (2009) i Spataro, Mullen, Burgess, Wells i Moss (2004) i depressió (per exemple, Lindert et al., 2013; Maniglio, 2009 i Pareda, 2010). També s'han trobat estudis que relacionen els abusos sexuals en la infantesa amb l'abús de substàncies (per exemple, Cutajar et al., 2010; Fergusson, Boden i Horwood, 2008), amb majors nivells de trastorn per estrès posttraumàtic, com es pot trobar en els estudis de Fergusson, McLeod i Horwood (2013) i de Pareda (2010), a més de treballs que parlen de trastorns psicòtics (per exemple, Cutajar et al., 2010; Spataro, Mullen, Burgess, Wells i Moss, 2004) i d'un augment de la ideació i dels intents de suïcidi (per exemple, Dinwiddie et al., 2000; Fergusson, McLeod i Horwood, 2013). Encara que aquestes siguen les consequiències psicopatològiques que més s'han observat en els estudis analitzats, se n'han pogut veure d'altres com ara el trastorn límit de la personalitat (Cutajar et al., 2010; Forouzan i Van Gijseghem, 2005; Hillberg, Hamilton i Dixon, 2011; Pareda, 2010), encara que com apareix en un estudi de Fossati, Madeddu i Meffei (1999), es creia en un primer moment que es trobarien més articles relacionats amb aquest trastorn psiquiàtric. També els trastorns dissociatius (Johnson, Pike i Chard, 2011).

Cal remarcar també que a diferència del que s'ha trobat en altres estudis que relacionen l'abús sexual infantil amb la fibromiàlgia (per exemple, LeónVega i Arias-Congrains, 2012; Boisset-Pioro; Esdaile i Fitzcharles, 1995), no s'ha trobat cap article que relacione l'ASI amb aquesta malaltia, per la qual cosa és necessària probablement una major investigació en aquest àmbit. Alguns dels estudis citats prèviament mostren que els infants víctimes d'abusos sexuals infantils presenten també majors nivells d'ansietat (Ruggiero, Mcleer i Dixon, 2000; Keyes et al., 2012; Hornor, 2010), de depressió (per exemple, Briere i Elliot, 1994; Ruggiero, Mcleer i Dixon, 2000), trastorn per estrès posttraumàtic (Ruggiero, Mcleer i Dixon, 2000; Briere i Elliot, 1994; Hornor, 2010) i ideació i intents de suïcidi (Briere i Elliot, 1994; Hornor, 2010). Llavors, és possible afirmar que hi ha una relació entre les psicopatologies que presenten les víctimes d'abusos sexuals infantils, a curt termini i a llarg termini i que aquestes no sols serien un fenomen immediat, després de l'abús, sinó que podrien aparèixer un temps després o no remetre quan apareixen en la infantesa. L'única conseqüència que no s'ha vist, en les víctimes infantils, ha estat l'abús d'alcohol i drogues, que sí que apareix en l'edat adulta, cosa que segurament es deu a l'accés restringit que tenen els menors a aquestes substàncies.

Per tant, cal posar de manifest que, com s'havia fet prèviament als estudis com el de Briere i Elliot (1994) i el de McCrae, Chapman i Christ (2006), en els treballs revisats tampoc no s'ha observat cap síndrome específica, com a resultat de l'abús sexual infantil, és a dir, l'abús sexual infantil pot donar lloc 
a nombroses i diverses psicopatologies tant a curt com a llarg termini, però no se n'ha trobat cap d'específica, que ocórrega després d'aquesta experiència. És possible comprovar que la prevalença d'aquest fenomen varia molt entre els estudis i és major en dones que en homes, però que a pesar d'això els nombres són elevats, per tal cal augmentar el nombre d'estudis, per a ambdós sexes i el d'estudis mixtos, però sobretot augmentar la investigació en homes, ja que aquesta ha estat més aviat escassa.

Cal ressaltar que la relació entre els abusos sexuals infantils i aquests psicopatologies no es poden entendre del tot de manera aïllada, ja que, a l'igual que hem vist quant a les psicopatologies que presenten les víctimes més joves, les que presenten els adults poden estar influïdes per diversos factors, a més, poden presentar-s'hi alguns de risc que empitjorarien el desenvolupament posterior. Apareix com a mediador l'aferrament adult i les relacions properes, els quals atenuen les consequiències psicopatològiques en els adults (Dimitrova et al., 2010; Forouzan i Van Gijseghem, 2005), i el suport social i familiar, que és de gran importància per a prevenir el desenvolupament de malalties mentals (Forouzan i Van Gijseghem, 2005; Sperry i Widom, 2013), de manera que els infants que tenen un adequat suport social i familiar presenten menys psicopatologies en l'edat adulta. També s'estudien les estratègies d'afrontament de les víctimes com a variable moduladora de l'abús sexual infantil en els estudis de Cantón i Cantón (2010), Cantón i Justicia (2008) i Steel, Sanna, Hammond, Whipple i Cross (2004), però s'ha trobat que són més una variable de risc, ja que només s'ha trobat que l'afrontament autodestructiu o evitador es desenvolupa en un pitjor ajust psicològic a llarg termini, i no hi ha una relació significativa entre l'afrontament aproximatiu i l'ajust posterior, cosa que requeriria un estudi més exhaustiu.

Finalment, alguns estudis com per exemple el de Chou (2012) inclou el gènere de la víctima com a mediador, però no apareix com a significativa la seua influència, és a dir, ser dona o ser home no condiciona les psicopatologies que es presenten. Com a variables de risc, és possible destacar sobretot les característiques de l'abús, com ara la gravetat, la freqüència, la duració, la relació amb l'agressor, el nombre d'agressors i l'ús de la violència, entre altres (per exemple, Bulik, Prescott i Kendler, 2001; Cantón i Cantón, 2010; Hillberg, Hamilton i Dixon, 2011), les quals indiquen que a major frequiència, duració, gravetat, en referència a la penetració o la falta de penetració, el major nombre d'abusadors, l'ús de violència i la familiaritat respecte a l'agressor, sobretot si és la figura paterna, augmenta el risc de presentar psicopatologies. També tenen importància les característiques sociodemogràfiques (per exemple, Chou, 2012; Pérez-Fuentes et al., 2013) i el funcionament familiar negatiu (per exemple, Briere i Elliott, 2003; Fergusson, Boden, i Horwood, 2008), entre altres. Cal destacar que no tots els estudis inclouen aquests tipus de variables a l'hora 
d'estudiar les conseqüències de l'abús sexual infantil, cosa que podria produir un biaix en els resultats que s'obtindrien, encara que hi ha moltes variables. Per això és important que els estudis futurs incloguen variables mediadores $\mathrm{i}$ de vulnerabilitat, cosa que ja està començant a fer-se (Cantón i Cantón, 2010; Cutajar et al., 2010).

Com s'ha pogut comprovar, l'abús sexual infantil no només afecta les víctimes en un termini curt de temps, les consequiències poden patir-se també a llarg termini, ja que és probable que aquestes no remeten fàcilment o que apareguen anys després que haja ocorregut l'agressió, encara que també és possible que no apareguen (McCrae, Chapman i Christ, 2006). Però a pesar que hi ha una minoria de infants que no presenten símptomes (Briere i Elliot, 1994), és important com a primera mesura d'actuació la prevenció de l'abús sexual infantil, que ajudaria a reduir l'aparició de malalties mentals en les víctimes d'aquests abusos. S'actuaria desenvolupant programes a les escoles (Redondo i Ortiz, 2005; Lalor i McEvaney, 2010) i als mitjans de comunicació, i amb teràpia amb els abusadors (Lalor i McEvaney, 2010), involucrant tant els infants com els adults en les tasques de prevenció (Zollner, Fuchs i Fegert, 2014), en les quals seria de molta importància la participació de professionals de la salut mental, com els psicòlegs.

Però, a pesar que és molt important la prevenció d'aquest fenomen, s'ha de fer també una detecció primerenca i una intervenció psicològica a les víctimes, tan aviat com siga possible perquè no arriben els problemes en l'edat adulta. I a més, cal que els infants aprenguen a detectar els abusos, ja que en algunes vegades els infants no són capaços de veure les situacions com abusives, i cal que aprenguen a demanar ajuda, ja que en moltes vegades els abusos es donen dins de la família (Finkelhor, 2005) i els infants no es veurien capaços de denunciar. El tractament no només ha d'incloure la víctima directa, també els familiars, sobretot els pares, ja que es pot donar una negació de l'abús, cosa que podria ser negativa per a la víctima, sensació de culpa dels pares, ràbia i sentiments de venjança, ansietat, depressió, baixa autoestima i deteriorament de les relacions familiars (Echeburúa i Guerricaechevarria, 2011), que com ja s'ha vist, són de molta importància per al bon desenvolupament de la víctima. A més, els equips han de ser multidisciplinari i ha d'haver-hi un psicòleg, entre altres professionals (Pou, Jordán i Gómez, 1996; Redondo i Ortiz, 2005).

A l'hora d'aplicar una teràpia psicològica s'ha de tenir en compte que els infants que han patit abusos sexuals són un grup heterogeni i de vegades és possible que no siga necessària una intervenció específica després de l'abús. A més, cal tenir present que hi ha diferents tipus de teràpies, cognitiva conductual -individual o en grup-, teràpia individual i familiar, i s'ha de tenir precaució a l'hora d'escollir la més adequada (Lalor i McEvaney, 2010). Però a pesar que, com s'esperava, s'ha trobat que els abusos sexuals infantils no només provoquen dificultats en la infantesa, sinó també en l'edat adulta i que apareixen variables 
i factors que tenen influència tant positiva com negativa en el desenvolupament posterior de la víctima, els resultats obtinguts presenten algunes limitacions que és important tenir en compte.

Una de les principals limitacions que s'ha de tenir en compte és que el disseny més emprat en els estudis és el retrospectiu, i a més els estudis que no empraven aquest tipus de disseny feien servir dades aportades per les víctimes, cosa que podria incloure falses memòries i oblits. Això podia donar lloc a falsos positius o a no detectar casos reals (Pareda, 2010). Altres problemes metodològics són els grups de control. Molts estudis no presenten grups de control adequats $i$ altres estudis ni tan sols en tenen, i no es poden comparar les víctimes amb la població general a causa, sobretot, del fet que les mostres provenien de diferents institucions i els autors coneixen les condicions de la mostra. Moltes vegades les mostres no són escollides a l'atzar entre la població, sinó que són expressament de registres oficials (per exemple, Bulik, Prescott i Kendler, 2001; Molnar, Buka i Kessler, 2001), i poden crear biaixos i resultats no vertaders en els estudis. Molts d'ells presenten mostres menudes, i és major el nombre d'estudis que se centren només en mostres de dones que en mostres d'homes, encara que ha Aquesta $t$ més elevat del que es podria esperar, el nombre d'estudis amb una mostra mixta (per exemple, Briere i Elliott, 2003; Fergusson, McLeod i Horwood, 2013; Young, Harford, Kinder i Savell, 2007), però el percentatge de dones és major que el d'homes.

Una dificultat que es troba és la de les múltiples definicions diferents d'abusos sexuals infantils, que varien d'un estudi a l'altre, cosa que comporta discrepàncies entre aquests (Fergusson, 1997; Briere i Elliot 1994) a l'hora de parlar de prevalença, psicopatologies, mediadors i variables de risc, entre altres. Per últim, encara que s'ha fet menció que la relació entre l'abús sexual infantil no siga directa sinó que hi ha altres variables que milloren o empitjoren el desenvolupament de la víctima, hi ha molts estudis que no inclouen aquests variables, cosa que produeix biaixos en els seus resultats. Per això, en investigacions futures és important que, en primer lloc, es tracte d'unificar els paràmetres per tal de definir els abusos sexuals infantils, i així dins d'aquesta s'inclourien els paràmetres. D'aquesta manera els resultats serien més generalitzables i no hi hauria tantes discrepàncies entre ells.

En segon lloc, caldria incloure-hi mostres d'un nombre més elevat de subjectes, i obtenir-les de la població general, no de mostres clíniques o universitàries, com s'ha donat en molts estudis, que no són representatives (Pareda, 2010). A més, hi ha molt poca investigació amb mostres masculines, ja que la majoria d'estudis utilitzen mostres de dones, per això, un estudi de Romano i De Luca (2001), posa de manifest que seria possible que hi haguera de tres a quatre vegades més casos que els que es coneixen, ja que es presta més atenció als abusos femenins, per tenir més risc, a més els homes revelen menys aques- 
tes situacions, per por a l'estigma homosexual o no interpretar la situació com abusiva. En tercer lloc, és important fer avaluacions més exhaustives sobre la credibilitat de les víctimes, ja que els estudis es basen en el que informen les víctimes, i seria possible que apareguera la síndrome de les falses memòries (Fergusson, 1997). És de gran dificultat establir el diagnòstic basant-nos quasi exclusivament en el testimoni de l'infant (Redondo i Ortiz, 2005), per això és important que el testimoni siga verídic. Per últim, és interessant que cada vegada més estudis incloguen variables de risc o mediadores entre l'abús sexual infantil, ja que és difícil entendre la relació de l'abús sexual infantil sense altres variables que influeixen (Cantón i Cantón, 2010; Cutajar et al., 2010).

També, fent referència a aquesta revisió, de cara a futures cerques o estudis, caldria tractar d'incloure més treballs, ja que els que s'hi han inclòs representen un percentatge molt menut dels estudis que es varen trobar en les cerques inicials (divuit de 27.035, 0,06\% del total), probablement perquè els criteris d'inclusió i exclusió eren massa restrictius i s'han pogut descartar treballs que haurien pogut aportar informació rellevant. A més, es podria fer una cerca centrada ara, d'una banda, en les psicopatologies que més han aparegut, i d'una altra, en les psicopatologies que s'esperava trobar i no ha estat el cas, com el trastorn límit de la personalitat i la fibromiàlgia. Per tant, és important que es continue investigant sobre aquest problema, ja que és de gran rellevància i les conseqüències d'aquestes conductes poden ser molt greus.

\section{Conclusions}

S'ha pogut comprovar que l'abús sexual infantil és un fenomen molt prevalent actualment $\mathrm{i}$ que ha anat augmentant l'interès en el seu estudi en els últims anys, probablement a causa d'una major consciència social, i s'ha arribat a donar una extensa publicació d'estudis i treballs sobre aquest problema, sobretot centrats en les psicopatologies que es desenvolupen arran d'aquesta experiència traumàtica, ja que és un fenomen que provoca múltiples i diverses malalties mentals, tant a curt com a llarg termini. Per això, és molt important, en primer lloc la prevenció, per conscienciar la societat i tractar d'evitar que ocórreguen, però com és una tasca difícil s'ha de fer un diagnòstic i una intervenció primerenca per pal-liar-ne tan aviat com siga possible les consequiències. A més, cal ensenyar els infants a identificar les situacions d'abús que poden viure, $\mathrm{i}$ denunciar-les, ja que algunes vegades els abusos sexuals infantils no es detecten perquè les víctimes no les identifiquen com a abús. Llavors, si s'augmenta la investigació sobre aquest problema, s'executen programes de prevenció i conscienciació, programes d'intervenció primerenca i detecció, es podran atenuar les seqüeles psicològiques que se'n poden derivar i millorar les condicions de vida de les víctimes. 


\section{Referències}

Berlinerblau, V. i Taylor, E. (2002). Abuso sexual: evaluación psiquiátrica forense en denuncias de niños. Cuadernos de Medicina Forense, 2, 33-42.

Briere, J. N. i Elliott, D. M. (1994). Immediate and long-term impacts of child sexual abuse. The Future of Children, 4, 54-69.

Briere, J. i Elliott, D.M. (2003). Prevalence and psychological sequelae of self-reported childhood physical and sexual abuse in a general population of men and women. Child Abuse and Neglect, 27, 1205-1222. doi:10.1016/j. chiabu.2003.09.008.

Boisset-Pioro, M.; Esdaile, J. M. i Fitzcharles, M. A. (1995). Sexual and physical abuse in women with fibromyalgia syndrome. Arthritis \& Rheumatism, 38, 235-241. doi:10.1002/art.1780380212.

Bulik, C. M.; Prescott, C. A. i Kendler, K. S. (2001). Features of childhood sexual abuse and the development of psychiatric and substance use disorders. The British Journal of Psychiatry, 179, 444-449. doi:10.1192/ bjp.179.5.444.

Calam, R.; Horne, L.; Glasgow, D. i Cox, A. (1998). Psychological disturbance and child sexual abuse: A followed-up study. Child Abuse and Neglect, 22, 901-913.

Cantón-Cortés, D. i Cantón, J. (2010). Coping with child sexual abuse among college students and post-traumatic stress disorder: The role of continuity of abuse and relationship with the perpetrator. Child Abuse and Neglect, 34, 496-506. doi:10.1016/j.chiabu.2009.11.004.

Cantón, D. i Justicia, F. (2008). Afrontamiento del abuso sexual infantil y ajuste psicológico a largo plazo. Psicothema, 20, 509-515.

Chou, K. L. (2012). Childhood sexual abuse and psychiatric disorders in middle-aged and older adults: evidence from the 2007 adult psychiatric morbidity survey. Journal of Clinical Psychiatry, 73, 1365-1371. doi:10.4088/ JCP.12m07946.

Cutajar, M. C.; Mullen, P. E.; Ogloff, J.; Thomas, S. D.; Wells, D. L. i Spataro, J. (2010). Psychopathology in a large cohort of sexually abused children followed up to 43 years. Child Abuse and Neglect, 34, 813-822. doi:10.1016/j. chiabu.2010.04.004.

Cutajar, M. C.; Mullen, P. E.; Ogloff, J. R.; Thomas, S. D.; Wells, D. L. i Spataro, J. (2010). Schizophrenia and other psychotic disorders in a cohort of sexually abused children. Archives of General Psychiatry, 67, 1114-1119. doi:10.1001/archgenpsychiatry.2010.147.

Dimitrova, N.; Pierrehumbert, B.; Glatz, N.; Torrisi, R.; Heinrichs, M.; Halfon, O.; et al. (2010). Closeness in relationships as a mediator between sexual abuse in childhood or adolescence and psychopathological out- 
come in adulthood. Clinical Psychology and Psychotherapy, 17, 183-195. doi:10.1002/cpp.650.

Dinwiddie, S.; Heath, A. C.; Dunne, M. P.; Bucholz, K. K.; Madden, P. A. F.; Slutske, W. S.; et al. (2000). Early sexual abuse and lifetime psychopathology: a co-twin-control study. Psychological Medicine, 30, 41-52.

Echeburúa, E. i Guerricaechevarría, C. (2011). Tratamiento psicológico de las víctimas de abuso sexual infantil intrafamiliar: un enfoque integrador. Behavioral Psychology/Psicología Conductual, 19, 469-486.

Fassler, I. R.; Amodeo, M.; Griffin, M. L.; Clay, C. M. i Ellis, M. A. (2005). Predicting long-term outcomes for women sexually abused in childhood: contribution of abuse severity versus family environment. Child Abuse and Neglect, 29, 269-284. doi:10.1016/j.chiabu.2004.12.006.

Fergusson, A. G. (1997). How good is the evidence relating to the frequency of childhood sexual abuse and the impact such abuse has on the lives of adult survivors? Public Health, 111, 387-391.

Fergusson, D. M.; McLeod, G. i Horwood, L. J. (2013). Childhood sexual abuse and adult developmental outcomes: findings from a 30-year longitudinal study in New Zealand. Child Abuse and Neglect, 37, 664-674. doi:10.1016/j.chiabu.2013.03.013.

Fergusson, D. M.; Boden, J. M. i Horwood, L. J. (2008). Exposure to childhood sexual and physical abuse and adjustment in early adulthood. Child Abuse and Neglect, 32, 607-619. doi:10.1016/j.chiabu.2006.12.018.

Finkelhor, D. (1991). Child Sexual Abuse. En M. L. Rosenberg i M. A. Fenley (Eds.), Violence in America. A public health approach (pp. 79-94). Nova York: Oxford University Press.

Finkelhor, D. (Ed.). (2005). Abuso sexual al menor. Causas, consecuencias y tratamiento psicosexual. Mèxic: Editorial Pax México.

Forouzan, E. i Van Gijseghem, H. (2005). Psychosocial adjustment and psychopathology of men sexually abused during childhood. International Journal of Offender Therapy and Comparative Criminology, 49, 626-651. doi:10.1177/0306624X04273650.

Fossati, A.; Madeddu, F. i Maffei, C. (1999). Borderline Personality Disorder and Childhood sexual abuse: a meta-analytic study. Journal Personality Disorders, 13, 268-280. doi:10.1521/pedi.1999.13.3.268.

Glasser, D. i Wiseman, M. (2000). Child Sexual Abuse. En E. Bittar i N. Bittar (Eds.), Biological Psychiatry (pp. 358-377). Aquesta ts Units: JAI Press INC.

García-Piña, C. A.; Loredo-Abdalá, A. i Gómez-Jiménez, M. (2009). Guía para la atención del abuso sexual infantil. Acta Pediátrica de México, 30, 94103. 
Hillberg, T.; Hamilton, C. i Dixon, L. (2011). Review of meta-analyses on the association between child sexual abuse and adult mental health difficulties: a systematic approach. Trauma, Violence and Abuse, 12, 38-49. doi:10.1177/1524838010386812.

Hornor, G. (2010). Child sexual abuse: Consequences and implications. Journal of Pediatric Health Care, 24, 358-364. doi:10.1016/j.pedhc.2009.07.003.

Johnson, D. M.; Pike, J. L. i Chard, K. M. (2011). Factors predicting PTSD, depression, and dissociative severity in female treatment-seeking childhood sexual abuse survivors. Child Abuse and Neglect, 25, 179-198. doi:10.1016/S0145-2134(00)00225-8.

Kendler, K. S.; Bulik, C. M.; Silberg, J.; Hettema, J. M.; Meyers, J. i Prescott, C. A. (2000). Childhood sexual abuse and adult psychiatric and substance use disorders in women. Archives of General Psychiatry, 57, 953-959. doi:10.1001/archpsyc.57.10.953.

Keyes, K. M.; Eaton, N. R.; Krueger, R. F.; McLaughlin, K. A.; Wall, M. M.; Grant, B. F.; et al. (2012). Childhood maltreatment and the structure of common psychiatric disorders. The British Journal of Psychiatry, 200, 107-115. doi:10.1192/bjp.bp.111.093062.

Lalor, K. i McElvaney, R. (2010). Child sexual abuse, links to later sexual explotation/high-risk sexual behavior, and prevention/treatment programs. Trauma, Violence and Abuse, 11, 159-177. doi:10.1177/1524838010378299.

León-Vega, S. i Arias-Congrains, J. (2012). Factores asociados al dolor en mujeres con fibromialgia. Revista de la Sociedad Peruana de Medicina Interna, 25, 173-182.

Lindert, J.; Ehrenstein, O. S.; Grashow, R.; Gal, G.; Braehler, E. i Weisskopf, M. G. (2013). Sexual and physical abuse in childhood is associated with depression and anxiety over the life course: systematic review and meta-analysis. International Journal of Public Health, 59, 359-372. doi:10.1007/ s00038-013-0519-5.

Maniglio, R. (2009). The impact of child sexual abuse on health. A systematic review of reviews. Clinical Psychology Review, 29, 647-657. doi:10.1016/j. cpr.2009.08.003.

McCrae, J. S.; Chapman, M. V. i Christ, S. L. (2006). Profile of children investigated for sexual abuse: Association with psychopathology symptoms and services. American Journal of Orthopsychiatry, 76, 468-481. doi:10.1037/0002-9432.76.4.468.

Molnar, B. E.; Buka, S. L. i Kessler, R. C. (2001). Child sexual abuse and subsequent psychopathology: results from the national comorbidity survey. American Journal of Public Health, 91, 753-760.

Murray, L. K.; Nguyen, A. i Cohen, J. A. (2014). Child sexual abuse. Child and Adolescent Psychiatric Clinics of North America, 23, 321-337. doi:10.1016/j.chc.2014.01.003. 
Pareda, N. (2010). Consecuencias psicológicas a largo plazo del abuso sexual infantil. Papeles del psicólogo, 31, 191-201.

Pérez-Fuentes, G.; Olfson, M.; Villegas, L.; Morcillo, C.; Wang, S. i Blanco, C. (2013). Prevalence and correlates of child sexual abuse: a national study. Comprehensive Psychiatry, 54, 16-27. doi:10.1016/j.comppsych. 2012.05.010.

Pou, J.; Jordán, I. i Gómez, L. (1996). Abuso sexual infantil. Nuestra experiencia de un año. Anales Españoles de Pediatría, 45, 245-248.

Romano, E. i De Luca, R. V. (2001). Male sexual abuse: a review of effects, abuse characteristics and links with later psychological functioning. Aggression and violent behavior, 6, 55-78. doi:10.1016/S1359-1789(99)00011-7.

Redondo, C. i Ortiz, M. R. (2005). El abuso sexual infantil. Boletín de la Sociedad de Pediatría de Asturias, Cantabria y Castilla y León, 45, 3-16.

Ruggiero, K. J.; McLeer, S. V. i Dixon, J. F. (2000). Sexual abuse characteristics associated with survivor psychopathology. Child Abuse and Neglect, 24, 951-964. doi:10.1016/S0145-2134(00)00144-7.

Spataro, J.; Mullen, P. E.; Burgess, P. M.; Wells, D. L. i Moss, S. A. (2004). Impact of Child Sexual Abuse on mental health: prospective study in males and females. The British Journal of Psychiatry, 184, 416-421. doi:10.1192/ bjp.184.5.416.

Sperry, D. M. i Widom, C. S. (2013). Child abuse and neglect, social support, and psychopathology in adulthood: A prospective investigation. Child Abuse and Neglect, 37, 415-425. doi:10.1016/j.chiabu.2013.02.006.

Steel, J.; Sanna, L.; Hammond, B.; Whipple, J. i Cross, H. (2004). Psychological sequelae of childhood sexual abuse: abuse-related characteristics, coping strategies, and atribucional style. Child Abuse and Neglect, 28, 785801. doi:10.1016/j.chiabu.2003.12.004.

Villagrá, P.; Fernández, P.; García-Vega, E. i González-Menéndez, A. (2013). Dual diagnosis in prisoners: childhood sexual and physical abuse as predictors in men and women. International Journal of Offender Therapy and Comparative Criminology, 20, 1-11. doi:10.1177/0306624X13513560.

Weiss, K. J. i Curcio, J. (2013). Sex, lies, and statistics: Inferences from the child sexual abuse accommodation syndrome. The Journal of the American Academy of Psychiatry and the Law, 41, 412-420.

Young, M. S.; Harford, K. L.; Kinder, B. i Savell, J. K. (2007). The relationship between childhood sexual abuse and adult mental health among undergraduates. Victim gender doesn't matter. Journal of Interpersonal Violence, 22, 1315-1331. doi: 10.1177/0886260507304552.

Zollner, H.; Fuchs, K. i Ferget, J. M. (2014). Prevention of sexual abuse: improved information is crucial.Child and Adolescent Psychiatry and Mental Health, 8, 1-9. 